\title{
Markov Dependence in Renewal Equations and Random Sums with Heavy Tails
}

\section{Søren Asmussen and Julie Thøgersen}





\title{
Markov Dependence in Renewal Equations and Random Sums with Heavy Tails
}

\author{
Søren Asmussen and Julie Thøgersen \\ Department of Mathematics \\ Aarhus University
}

\begin{abstract}
The Markov renewal equation

$$
Z_{i}(x)=z_{i}(x)+\sum_{j \in \mathcal{E}} \int_{0}^{x} Z_{j}(x-y) F_{i j}(\mathrm{~d} y), \quad i \in \mathcal{E},
$$

is considered in the subcritical case where the matrix of total masses of the $F_{i j}$ has spectral radius strictly less than one, and the asymptotics of the $Z_{i}(x)$ is found in the heavy-tailed case involving a local subexponential assumption on the $F_{i j}$. Three cases occur according to the balance between the $z_{i}(x)$ and the tails of the $F_{i j}$, A crucial step in the analysis is obtaining multivariate and local versions of a lemma due to Kesten on domination of subexponential tails. These also lead to various results on tail asymptotics of sums of a random number of heavy-tailed random variables in models which are more general than in the literature.
\end{abstract}

\section{Introduction}

The occurrence of heavy tails has been argued repeatedly in a variety of application areas covering insurance and finance ([1]), telecommunications and internet traffic ([2]), optics ([3]), cell proliferation ([4]) and many more; a broad overview is in [5]. Correspondingly, performance analysis of models for such situations has triggered a vast literature on probabilistic features of heavy tails.

This paper deals with two particular problems in this area. The first is asymptotics of renewal-type equations of the form

$$
Z_{i}(x)=z_{i}(x)+\sum_{j \in \mathcal{E}} \int_{0}^{x} Z_{j}(x-y) F_{i j}(\mathrm{~d} y), \quad i \in \mathcal{E},
$$

where $\mathcal{E}$ is a finite index set, $\left(Z_{i}\right)_{i \in E}$ a set of unknown functions defined on $[0, \infty)$, $\left(z_{i}\right)_{i \in \mathcal{E}}$ a set of non-negative known functions, and $\left(F_{i j}\right)_{i, j \in \mathcal{E}}$ a set of non-negative heavy-tailed measures on $[0, \infty)$. The second is the tail behaviour of a random sum

$$
S=\sum_{i=1}^{N} X_{i}
$$


where $N \in \mathbb{N}$ is a light-tailed r.v. and the $X_{i}$ are non-negative heavy-tailed r.v.'s, such that certain types of dependence to be specified later may occur.

The simple renewal equation

$$
Z(x)=z(x)+\int_{0}^{x} Z(x-y) F(\mathrm{~d} y)
$$

is a classical structure in applied probability and occurs for example in branching processes ([6]), ruin problems ([7]) and ergodicity problems for possibly nonMarkovian processes ([8, VI.1]). The emphasis is usually on asymptotic properties of $Z(x)$ as $x \rightarrow \infty$ where the simplest situation is existence of a limit ${ }^{1}$ when $F$ is a probability measure, i.e. when $\|F\|=1$ where $\|F\|$ is the total mass of $F$. However, in the branching process example $\|F\|$ is the expected number of children of an individual so obviously the case $\|F\| \neq 1$ is of interest. One then typically has an exponential order $\mathrm{e}^{\gamma x}$ of $Z(x)$. Here $\gamma>0$ when $\|F\|>1$ and $\gamma<0$ when $\|F\|<1$ and $F$ is light-tailed (this last situation also occurs in Cramér-Lundberg asymptotics for ruin probabilities and queues, cf. [8, V.7]). For $\|F\|<1$ and $F$ heavy-tailed, the order depends on a delicate balance between the tails of $F$ and $z$ and in fact the results are more recent, [9] (see also [10] for a closely related results).

The system (1.1) goes under the name of the Markov renewal equation, cf. [8]; this terminology stems from the case of

$$
\boldsymbol{P}=\left(\left\|F_{i j}\right\|\right)_{i, j \in \mathcal{E}}
$$

being stochastic, i.e. the transition matrix of a Markov chain $\xi_{0}, \xi_{1}, \ldots$ In branching processes, it occurs when individuals have several types, and in insurance and finance, it relates to regime-switching; another relevant example comes from computer reliability problems, [11]. The known asymptotic results on the $Z_{i}(x)$ depend crucially on the spectral radius $\rho$ of $\boldsymbol{P}$ : if $\rho=1$, in particular if $\boldsymbol{P}$ is stochastic, limits exists whereas otherwise the order is exponential, $\mathrm{e}^{\gamma x}$, where $\gamma>0$ when $\rho>1$ and $\gamma<0$ when $\rho<1$ and the $F_{i j}$ are light-tailed [again, regularity conditions are required]. The gap is the case $\rho<1$ and heavy tails. The main contribution of the paper in the setting of (1.1) is to fill this gap. The result is stated as Theorem 2.2 below. Three cases occur depending on whether the tail of the $z_{i}$ or the $F_{i j}$ dominate, or if they are of same order. The conditions involve the non-standard concept of local subexponentiality.

One main example of random sums like (1.2) is total claims distributions in insurance: $N$ is the number of claims in a given period and $X_{1}, X_{2}, \ldots$ the claim sizes which classically are taken i.i.d. and independent of $N$ which is assumed lighttailed (for example, the negative binomial distribution is popular because of its interpretation as a gamma mixture of Poissons). In finance, $S$ could be the payoff of a portfolio consisting of assets with values $X_{1}, X_{2}, \ldots$ With light-tailed $X_{i}, \mathbb{P}(S>x)$ decays roughly exponential, with heavy tails the asymptotic form is $\mathbb{E} N \cdot \mathbb{P}\left(X_{1}>x\right)$. Our main result in that direction, Theorem 2.3 below, is an extension to sums of the form $\sum_{k=1}^{d} \sum_{1}^{N_{k}} X_{i ; k}$ where $N_{1}, \ldots, N_{d}$ are dependent and the distribution of

\footnotetext{
${ }^{1}$ This and the following statements require regularity conditions which we omit; see the citations given
} 
$X_{i ; k}$ depends on $i$. For example in the insurance setting, $\left(N_{1}, \ldots, N_{d}\right)$ could be conditionally independent given $\left(\tau_{1}, \ldots, \tau_{d}\right)$ with Poisson rate $\lambda_{k}$ for $N_{k}$, where $\tau_{k}$ is the time spent by some environmental process in state $k$ in the period $[0, T]$ (dependence occurs because $\tau_{1}+\cdots+\tau_{d}=T$ ). The main step in the proof of Theorem 2.3 is a version of a classical lemma due to Kesten on tail domination of subexponential sums; for the proof of Theorem 2.2 we also need a local version of this.

The paper is organised as follows. Section 2 starts with some necessary background, in particular on local subexponentiality, and proceeds to state the two main results of the paper, Theorems 2.2 and 2.3 referred to above. The rest of the paper is then proofs supplemented with miscellaneous results of some independent interest. Sections 3-5 give the random sum part in various settings. This together with a number of additional steps then allows to conclude the proof of Theorem $2.2 \mathrm{in}$ Section 6. This proceeds by first assuming $\boldsymbol{P}$ to be substochastic, thereby allowing Markov chain interpretations, and finally reduce the general case to this by a Perron-Frobenius type transformation.

\section{Preliminaries and statement of main results}

For a distribution $F$ on $\mathbb{R}^{+}$, let $F(x)=F(0, x]$ be the distribution function (c.d.f.) and $\bar{F}(x)=1-F(x)=F(x, \infty)$ the tail. If $F$ and $G$ are distributions and $X \sim F$ and $Y \sim G$ independent random variables, then $F * G$ denotes the convolution of $F$ and $G$,

$$
F * G(x)=\int_{0}^{x} G(x-y) F(d y)=\int_{0}^{x} F(x-y) G(d y),
$$

which is the distribution of the sum $X+Y$.

We next briefly mention the most standard definitions and relations in the heavytailed area. For a more detailed and thorough treatment, see [1], [12]. A distribution $F$ is said to be heavy-tailed if $F$ does not possess any positive exponential moments, that is $\int_{-\infty}^{\infty} \exp (\lambda x) F(\mathrm{~d} x)=\infty$ for all $\lambda>0$. It is long-tailed, written $F \in \mathcal{L}$, if it has unbounded support and $\bar{F}(x+y) / \bar{F}(x) \rightarrow 1$ as $x \rightarrow \infty$ for any fixed $y \in \mathbb{R}$. Any $F \in \mathcal{L}$ is especially heavy-tailed. $F$ is said to be subexponential if $\overline{F^{* n}}(x) / \bar{F}(x) \rightarrow n$ for all $n$ (actually, it is sufficient that this holds for $n=2$ ). The intuition is that the only significant way the sum of independent $F$-distributed random variables can exceed som large threshold $x$ is if the maximum of the random variables exceeds that $x$. This is also known as the principle of a single big jump. Similarly, a density $f$ is long-tailed if $f(x)>0$ for all sufficiently large $x$ and $f(x+t) / f(x) \rightarrow 1$ for any fixed $t$. If $F$ and $G$ have densities $f, g$, respectively, the convolution $F * G$ then has density $f * g$ given by $f * g(x)=\int_{0}^{x} f(x-y) g(y) \mathrm{d} y$. The density $f$ of $F$ is said to be subexponential, written $F \in \mathcal{S}_{a c}$, if $f$ is long-tailed and $f^{* 2}(x)=$ $\int_{0}^{x} f(x-y) f(y) d y \sim 2 f(x)$ as $x \rightarrow \infty$. Note that $\mathcal{S}_{a c}$ is a subclass of $\mathcal{S}$. Also, since subexponentiality is a tail property it is actually sufficient for a distribution $F$ to only have a density $f(x)$ for sufficiently large $x$.

We proceed to the less standard concepts of local long-tailedness and subexponentiality, as introduced in [9]. The local property can be viewed as intermediate 
between being long-tailed (subexponential) and having a long-tailed (subexponential) density. First, we need to introduce some notation. For a fixed $T>0$ define $\Delta=\Delta_{T}=(0, T]$ and let $x+\Delta=\{x+y \mid y \in \Delta\}=(x, x+T]$.

Definition 2.1. A distribution $F$ is said to be $\Delta$-long-tailed, written $F \in \mathcal{L}_{\Delta}$, if $F(x+\Delta)>0$ for all sufficiently large $x$ and $F(x+y+\Delta) / F(x+\Delta) \rightarrow 1$ as $x \rightarrow \infty$ for any fixed $y$. It is is $\Delta$-subexponential, written $F \in \mathcal{S}_{\Delta}$, if $F \in \mathcal{L}_{\Delta}$ and $F^{* 2}(x+\Delta) / F(x+\Delta) \rightarrow 2$.

Notice that if we allow $T=\infty$ then the class $\mathcal{L}_{\Delta}$ corresponds to the ordinary longtailed distributions $\mathcal{L}$; if $T<\infty$, then $\mathcal{L}_{\Delta} \subset \mathcal{L}$. Similarly, for any finite $T$ the class $\mathcal{S}_{\Delta} \subset \mathcal{S}$, and if we allow $T=\infty$ the two classes coincides. It appears that $\Delta$-subexponential distributions possess many similar properties as the ordinary subexponential distributions. A main and crucial difference is that the tail function $\bar{F}(x)$ is monotone whereas $F(x+\Delta)$ may not be. Also, it it worth noticing that a distribution $F$ with a subexponential density $f$ is $\Delta$-subexponential for any $T>0$.

We are now ready to state our main results. Let $\boldsymbol{P}$ be the the matrix with $i j$ th element $p_{i j}=\left\|F_{i j}\right\|$.

Theorem 2.2. Consider the Markov renewal equation (1.1). Assume that $\boldsymbol{P}$ is irreducible with $\operatorname{spr}(\boldsymbol{P})<1$ and that $F_{i j}(x+\Delta) \sim p_{i j} c_{i j} G(x+\Delta)$ where $c_{i j}>0$ and $G$ is a $\Delta$-subexponential distribution function for all $T>0$. Let $g(x)=G(x, x+1)$, $I_{j}=\int_{0}^{\infty} z_{j}(y) \mathrm{d} y$, and let $d_{i j}$ be the ijth element of the matrix $(\boldsymbol{I}-\boldsymbol{P})^{-1} \boldsymbol{M}(\boldsymbol{I}-\boldsymbol{P})^{-1}$ with $\boldsymbol{M}=\left(p_{k \ell} c_{k \ell}\right)_{k, \ell \in \mathcal{E}}, k_{i j}$ the ijth element of $\sum_{0}^{\infty} \boldsymbol{P}^{n}=(\boldsymbol{I}-\boldsymbol{P})^{-1}$. Then three cases occur:

i) Assume that $z_{j}$ is directly Riemann integrable and $z_{j}(x) / g(x) \rightarrow 0$ for all $j \in \mathcal{E}$. Then

$$
Z_{i}(x) \sim \sum_{j \in \mathcal{E}} I_{j} d_{i j} g(x)
$$

ii) Assume that $z_{j}$ is directly Riemann integrable and $z_{j}(x) / g(x) \rightarrow a_{j}$ where $a_{j}>0$ for at least one $j \in \mathcal{E}$. Then

$$
Z_{i}(x) \sim \sum_{j \in \mathcal{E}}\left(I_{j} d_{i j}+k_{i j} a_{j}\right) g(x)
$$

iii) Assume that $z_{j}(y) / I_{j}$ has a subexponential density and that $z_{j}(x) / g(x) \rightarrow \infty$ for all $j$. Then

$$
Z_{i}(x) \sim \sum_{j \in \mathcal{E}} k_{i j} z_{j}(x)
$$

Theorem 2.3. Let $N_{1}, \ldots, N_{d}$ be non-negative integer-valued r.v.'s and

$$
S=\sum_{i=1}^{d} \sum_{j=1}^{N_{i}} X_{i j}
$$


where the $X_{i j}$ are independent given $N_{1}, \ldots, N_{d}$ with distribution $F_{i}$ of $X_{i j}$. Assume $\bar{F}_{i}(x) \sim c_{i} \bar{F}(x)$ for some $F \in \mathcal{S}$ and some $c_{1}, \ldots, c_{d} \geq 0$ and that there are $\varepsilon_{i}>0$ such that $\mathbb{E}\left[\left(1+\varepsilon_{i}\right)^{N_{i}}\right]<\infty$ for all $i=1, \ldots, d$. Then

$$
\mathbb{P}(S>x) \sim\left(c_{1} \mathbb{E}\left[N_{1}\right]+\cdots+c_{d} \mathbb{E}\left[N_{d}\right]\right) \bar{F}(x) .
$$

Note that $N_{1}, \ldots, N_{d}$ are not assumed independent. A local version of Theorem 2.3 is given below as Theorem 4.3.

\section{Random sums of subexponentials}

A classical bound due to Kesten ([13] pp. 148-9, [1] p. 41) states that for a subexponential distribution $F_{0}$ and $\varepsilon>0$ there exists a $D_{0}=D_{0}(\varepsilon)>0$

$$
\overline{F_{0}^{* n}}(x) \leq D_{0}(1+\varepsilon)^{n} \bar{F}_{0}(x) \quad \text { for any } x \geq 0 \text { and } n \in \mathbb{N} .
$$

The following result is a similar result involving convolution of the tails of multiple subexponential distribution functions.

Proposition 3.1. Let $F \in \mathcal{S}$, let $c_{1}, \ldots, c_{d} \geq 0$ and let $F_{1}, \ldots, F_{d}$ be distributions with $\bar{F}_{i}(x) \sim c_{i} \bar{F}(x)$. Then for every $\varepsilon>0$ there exists a $D=D(\varepsilon)$ such that the following inequality holds

$$
\overline{F_{1}^{* n_{1}} * \cdots * F_{d}^{* n_{d}}}(x) \leq D(1+\varepsilon)^{n} \bar{F}(x) \quad \text { for all } x \geq 0 \text { and } n_{1}, \ldots, n_{d} \in \mathbb{N}
$$

where $n=n_{1}+\ldots+n_{d}$. Furthermore

$$
\overline{F_{1}^{* n_{1}} * \cdots * F_{d}^{* n_{d}}}(x) \sim\left(c_{1} n_{1}+\cdots+c_{d} n_{d}\right) \bar{F}(x) .
$$

Proof. Define the distribution function $F_{0}$ by having tail

$$
\bar{F}_{0}(x)=\sup _{i=1, \ldots, d}\left\{\bar{F}_{i}(x)\right\} \sim \sup _{i=1, \ldots, d}\left\{c_{i} \bar{F}(x)\right\}=\sup _{i=1, \ldots, d}\left\{c_{i}\right\} \bar{F}(x) .
$$

Notice that taking the supremum will preserve properties as being cadlag, decreasing, and having values between $[0,1]$. Since the tail of $F_{0}$ is the supremum of the tails of some c.d.f.'s, $F_{0}$ is indeed af c.d.f. itself. Let $k=\sup _{i=1, \ldots, d} c_{i}$. The tail of $F_{0}$ is then asymptotically of the form $k \bar{F}(x)$, and since $\mathcal{S}$ is closed under tail equivalence we must have $F_{0} \in \mathcal{S}$. From the classical Kesten bound it follows that for every $\varepsilon>0$ there is a $D_{0}=D_{0}(\varepsilon)$ such that $(3.1)$ holds. Now for $i=0,1 \ldots, d$ let

$$
X_{i, 1}, \ldots, X_{i, n_{i}} \sim F_{i} \quad \text { where } n_{0}=n
$$

Then by construction we have the stochastic ordering

$$
X_{i, j} \leq_{s t} \quad X_{0, k} \quad \text { for all } i=1, \ldots, d, j=1, \ldots, n_{i}, k=1, \ldots, n,
$$

which implies that the sums also have the corresponding stochastic ordering

$$
X_{1,1}+\cdots+X_{1, n_{1}}+\cdots+X_{d, 1}+\cdots+X_{d, n_{d}} \leq_{s t} X_{0,1}+\cdots+X_{0, n} .
$$


Written in terms of convolutions of the tails of the distribution functions this means that

$$
\overline{F_{1}^{* n_{1}} * \cdots * F_{d}^{* n_{d}}}(x) \leq \overline{F_{0}^{* n}}(x) .
$$

By (3.1) it is therefore sufficient to show that there exists a $D=D(\varepsilon)$ such that

$$
D_{0} \overline{F_{0}}(x) \leq D \bar{F}(x)
$$

From the definition of $F_{0}$ and $\bar{F}_{i}(x) \sim c_{i} \bar{F}(x)$ it follows that whenever $K>$ $\max _{1, \ldots, d}\left\{c_{i}\right\}$, there exists a $x_{0} \geq 0$ such that $\overline{F_{0}}(x) / \bar{F}(x) \leq K$ for all $x>x_{0}$. Replacing $K$ by a larger $K$ if necessary, we may assume this holds for all $x$. Thus

$$
\begin{aligned}
D_{0} \overline{F_{0}}(x) & =D_{0} \frac{\overline{F_{0}}(x)}{\bar{F}(x)} \bar{F}(x) \leq D_{0} \sup _{x}\left\{\frac{\overline{F_{0}}(x)}{\bar{F}(x)}\right\} \bar{F}(x) \\
& \leq D_{0}\left(\sup _{x \leq x_{0}}\left\{\frac{\overline{F_{0}}(x)}{\bar{F}(x)}\right\}+\sup _{x>x_{0}}\left\{\frac{\overline{F_{0}}(x)}{\bar{F}(x)}\right\}\right) \bar{F}(x) \\
& \leq D_{0}\left(K+2 \max _{i=1, \ldots, d}\left\{c_{i}\right\}\right) \bar{F}(x) \equiv D \bar{F}(x)
\end{aligned}
$$

with $D=D_{0}\left(K+2 \max _{i=1, \ldots, d}\left\{c_{i}\right\}\right)$. The asympotics in (3.3) now easily follows. To this end, just notice that $\overline{F_{i}^{* n_{i}}}(x) \sim n_{i} c_{i} \bar{F}(x)$ for all $i$ by standard subexponential theory and proceed by induction, using that $\overline{G_{1} * G_{2}}(x) \sim\left(\gamma_{1}+\gamma_{2}\right) \bar{F}(x)$ if $\bar{G}_{i}(x) \sim$ $\gamma_{i} \bar{F}(x)$ for $i=1,2$ and $F \in \mathcal{S}$.

Kesten's bounds is commonly used as majorant in dominated convergence to find the asymptotics of a randomly stopped sum of i.i.d. subexponential random variables. Proposition 3.1 can be used correspondingly for the multidimensional case which we use to give the proof of Theorem 2

Proof. The law of total probability yields

$$
\begin{aligned}
\frac{\mathbb{P}(S>x)}{\bar{F}(x)} & =\sum_{n_{1}, n_{2}, \ldots, n_{d}=1}^{\infty} \mathbb{P}\left(N_{1}=n_{1}, \ldots, N_{d}=n_{d}\right) \frac{\overline{F^{* n_{1}} * \cdots * F^{* n_{d}}}(x)}{\bar{F}(x)} \\
& \rightarrow \sum_{n_{1}, n_{2}, \ldots, n_{d}=1}^{\infty} \mathbb{P}\left(N_{1}=n_{1}, \ldots, N_{d}=n_{d}\right)\left(c_{1} n_{1}+\cdots+c_{d} n_{d}\right) \\
& =c_{1} \mathbb{E}\left[N_{1}\right]+\cdots+c_{d} \mathbb{E}\left[N_{d}\right]
\end{aligned}
$$

using Proposition 3.1 and dominated convergence; this is justified since Hölder's inequality implies that $\mathbb{E}\left[(1+\varepsilon)^{N_{1}+\cdots+N_{d}}\right]<\infty$ if we take (say) $1+\varepsilon=\min \left\{1+\varepsilon_{1}\right.$, $\left.\ldots, 1+\varepsilon_{d}\right\}^{1 / d}$.

\section{Random sums of local subexponentials}

The objective of the following Lemma is to obtain an upper bound for convolution of local subexponential distribution functions, which is needed to expand the local version of Kesten's bounds stated as Proposition 4 in [9] to include the convolution of several local subexponential distribution functions. 
Lemma 4.1. Let $H \in \mathcal{S}_{\Delta}$. Assume that $G_{i}, i=1,2$ are distributions satisfying

$$
G_{i}(x+\Delta) \leq b_{i} H(x+\Delta) \quad \text { for all } x \geq x_{0}
$$

for some $b_{i} \geq 1$ and $x_{0}$. Then for some constant a depending only on $H$, it holds that

$$
G_{1} * G_{2}(x+\Delta) \leq a b_{1} b_{2} H(x+\Delta) \quad \text { for all } x \geq x_{0} .
$$

Proof. Define

$$
a_{1}=\sup _{u, v \geq x_{0}:|u-v| \leq 1} \frac{H(u+\Delta)}{H(v+\Delta)}
$$

and notice that $a_{1}$ is finite for sufficiently large $x_{0}$ since $H$ is $\Delta$-subexponential, and therefore especially $\Delta$-longtailed. We have the representation

$$
\begin{aligned}
G_{1} * G_{2}(x+\Delta) & =\int_{0}^{x} G_{2}(x-y+\Delta) G_{1}(\mathrm{~d} y)+\int_{x}^{x+T} G_{2}(0, x+T-y] G_{1}(\mathrm{~d} y) \\
& \equiv P_{1}(x)+P_{2}(x) .
\end{aligned}
$$

Consider the first term. Let $k$ be the smallest integer such that $x / k \leq 1$ and $1 / k \leq T$. Split interval $(0, x]$ into $k$ disjoint equally sized parts. For $x \geq x_{0}$ the first term can then be written as a sum and assessed as follows

$$
\begin{aligned}
P_{1}(x) & =\sum_{i=0}^{k-1} \int_{x i / k}^{x(i+1) / k} G_{2}(x-y+\Delta) G_{1}(\mathrm{~d} y) \\
& \leq b_{2} \sum_{i=0}^{k-1} \int_{x i / k}^{x(i+1) / k} H(x-y+\Delta) G_{1}(\mathrm{~d} y) \\
& \leq a_{1} b_{2} \sum_{i=0}^{k-1} \int_{x i / k}^{x(i+1) / k} H(x-x i / k+\Delta) G_{1}(\mathrm{~d} y) \\
& \leq a_{1} b_{2} \sum_{i=0}^{k-1} H(x-x i / k+\Delta) G_{1}(x i / k, x(i+1) / k) \\
& \leq a_{1} b_{1} b_{2} \sum_{i=0}^{k-1} H(x-x i / k+\Delta) H(x i / k+\Delta)
\end{aligned}
$$

where the summands can be evaluated backwards

$$
\begin{aligned}
\int_{x i / k}^{x(i+1) / k} H(x-y+\Delta) H(\mathrm{~d} y) & \geq \frac{1}{a_{1}} \int_{x i / k}^{x(i+1) / k} H(x-i x / k+\Delta) H(\mathrm{~d} y) \\
& =\frac{1}{a_{1}} H(x-i x / k+\Delta) H(x i / k+\Delta) .
\end{aligned}
$$

Inserting this upper bound for the summands yields the inequality

$$
\begin{aligned}
P_{1}(x) & \leq a_{1}^{2} b_{1} b_{2} \sum_{i=0}^{k-1} \int_{x i / k}^{x(i+1) / k} H(x-y+\Delta) H(\mathrm{~d} y) \\
& =a_{1}^{2} b_{1} b_{2} \int_{0}^{x} H(x-y+\Delta) H(\mathrm{~d} y) \\
& \leq a_{1}^{2} b_{1} b_{2} H^{* 2}(x+\Delta) .
\end{aligned}
$$


Since $H$ is $\Delta$-subexponential there must be a finite $\delta$ such that

$$
H^{* 2}(x+\Delta) \leq(2+\delta) H(x+\Delta) \quad \text { for all } x \geq x_{0}
$$

if $x_{0}$ is sufficiently large. This provides the final inequality for the first term

$$
P_{1}(x) \leq(2+\delta) a_{1}^{2} b_{1} b_{2} H(x+\Delta) .
$$

Now, consider the second term. It follows directly that

$$
P_{2}(x) \leq \int_{x}^{x+T} G_{1}(\mathrm{~d} y) \leq b_{1} H(x+\Delta) \leq b_{1} b_{2} H(x+\Delta) .
$$

Altogether we now have

$$
G_{1} * G_{2}(x+\Delta) \leq\left((2+\delta) a_{1}^{2}+1\right) b_{1} b_{2} H(x+\Delta)
$$

so we can take $a=(2+\delta) a_{1}^{2}+1$.

We can use this to obtain a local version of Proposition 3.1.

Proposition 4.2. Let $F \in \mathcal{S}_{\Delta}$ for some $\Delta$, and $F_{i}(x+\Delta) \sim c_{i} F(x+\Delta)$ for $c_{1}, \ldots, c_{d} \geq 0$. Then for every $\varepsilon>0$ there exists a $D=D(\varepsilon)$ and $a x_{0}=x_{0}(\varepsilon)$ such that the following inequality holds

$$
F_{1}^{* n_{1}} * \cdots * F_{d}^{* n_{d}}(x+\Delta) \leq D(1+\varepsilon)^{n} F(x+\Delta)
$$

for all $x \geq x_{0}$ and $n_{1}, \ldots, n_{d} \in \mathbb{N}$ where $n=n_{1}+\ldots+n_{d}$. Furthermore

$$
F_{1}^{* n_{1}} * \cdots * F_{d}^{* n_{d}}(x+\Delta) \sim\left(c_{1} n_{1}+\cdots+c_{d} n_{d}\right) F(x+\Delta) .
$$

Proof. Let $\varepsilon>0$ be given. Notice that from Proposition 1 it follows that for $i=$ $1, \ldots, d$ there is a $V_{i}=V_{i}(\varepsilon)$ such that

$$
F_{i}^{* n_{i}}(x+\Delta) \leq V_{i}(1+\varepsilon)^{n_{i}} F(x+\Delta) .
$$

(4.2) can then be proven by induction using Lemma 4.1. For $d=2$ it follows directly from the Lemma that there is an a such that

$$
F_{1}^{* n_{1}}(x+\Delta) * F_{2}^{* n_{2}}(x+\Delta) \leq a V_{1} V_{2}(1+\varepsilon)^{n_{1}+n_{2}} F(x+\Delta) .
$$

Letting $D=a V_{1} V_{2}$ we have the desired inequality. Assume that (4.2) holds for $d$ distribution functions with constant $D$. Now consider the case with $d+1$ distribution functions, then we have

$$
\begin{aligned}
F_{1}^{* n_{1}} * \cdots * F_{d+1}^{* n_{d+1}}(x+\Delta) & =\left(F_{1}^{* n_{1}} * \cdots * F_{1}^{* n_{d}}\right) * F_{d+1}^{* n_{d+1}}(x+\Delta) \\
& \leq a D V_{d+1}(1+\varepsilon)^{n_{1}+\cdots+n_{d}}(1+\varepsilon)^{n_{d+1}} F(x+\Delta) \\
& =a D V_{d+1}(1+\varepsilon)^{n_{1}+\cdots+n_{d+1}} F(x+\Delta) .
\end{aligned}
$$

From Corollary 2 in [9] it follows that $F_{i}^{* n_{i}}(x+\Delta) \sim n_{i} c_{i} F(x+\Delta)$ for $i=1, \ldots, d$, and using Proposition 3 in [9] one can further deduce that

$$
F_{1}^{* n_{1}} * \cdots * F_{d}^{* n_{d}}(x+\Delta) \sim\left(n_{1} c_{1}+\cdots+n_{d} c_{d}\right) F(x+\Delta) .
$$

Theorem 4.3. Assume in addition to the ssumptions of Theorem 2.3 that $F \in \mathcal{S}_{\Delta}$ for some $\Delta$ and $F_{i}(x+\Delta) \sim c_{i} F(x+\Delta)$ for $c_{1}, \ldots, c_{d} \geq 0$. Then

$$
F^{* N_{1}} * \cdots * F^{* N_{d}}(x+\Delta) \sim\left(c_{1} \mathbb{E}\left[N_{1}\right]+\cdots+c_{d} \mathbb{E}\left[N_{d}\right]\right) F(x+\Delta) .
$$

Proof. Use dominated convergence justified by Proposition 4.2. 


\section{$5 \quad$ Random sums with subexponential densities}

In [9] it is shown that when $F \in \mathcal{S}_{a c}$ then, for any $\varepsilon>0$, there exist $x_{0}=x_{0}(\varepsilon)$ and $V=V(\varepsilon)$ such that

$$
f^{* n}(x) \leq V(1+\varepsilon)^{n} f(x) \quad \text { for any } x \geq x_{0} \text { and } n \in \mathbb{N} .
$$

We now seek to obtain a version of Theorem 2.3 involving densities instead. To do this we need an upper bound for convolutions, as for the local subexponential case.

Lemma 5.1. Let $F \in \mathcal{S}_{a c}$. Let $f_{1}$ and $f_{2}$ be densities with the properties

$$
f_{i}(x) \leq b_{i} f(x) \quad \text { with } b_{i}>1 \text { for } i=1,2 \text { and all } x \geq x_{0} .
$$

Then there is a constant $A$ independent of $f_{1}$ and $f_{2}$ such that

$$
f_{1} * f_{2}(x) \leq A b_{1} b_{2} f(x) \quad \text { for all } x \geq x_{0} .
$$

Proof. Since $f$ is longtailed then $f(x)>0$ for all $x \geq x_{0}$ for $x_{0}$ sufficiently large, hence

$$
a \equiv \sup _{y \in\left(0, x-x_{0}\right)} \frac{f(x-y)}{f(x)}
$$

is finite. Consider the partition

$$
\begin{aligned}
f_{1} * & f_{2}(x)=\int_{0}^{x} f_{1}(x-y) f_{2}(y) \mathrm{d} y \\
& =\int_{0}^{x-x_{0}} f_{1}(x-y) f_{2}(y) \mathrm{d} y+\int_{x-x_{0}}^{x_{0}} f_{1}(x-y) f_{2}(y) \mathrm{d} y+\int_{x_{0}}^{x} f_{1}(x-y) f_{2}(\mathrm{~d} y) \\
& \equiv I_{1}+I_{2}+I_{3} .
\end{aligned}
$$

Now each term is assessed individually, starting with the first for $x \geq x_{0}$,

$$
I_{1} \leq b_{1} \int_{0}^{x-x_{0}} f(x-y) F_{2}(\mathrm{~d} y) \leq a b_{1} \int_{0}^{x-x_{0}} f(x) F_{2}(\mathrm{~d} y) \leq a b_{1} b_{2} f(x) .
$$

Analogously for the third term,

$$
I_{3}=\int_{0}^{x-x_{0}} f_{2}(x-y) f_{1}(y) \mathrm{d} y \leq a b_{1} b_{2} f(x) .
$$

Only the second term is left to be evaluated. Now let $x \geq 2 x_{0}$, then it follows

$$
I_{2}=b_{1} b_{2} \int_{x-x_{0}}^{x_{0}} f(x-y) f(y) \mathrm{d} y \leq b_{1} b_{2} \int_{0}^{x} f(x-y) f(y) \mathrm{d} y=b_{1} b_{2} f^{* 2}(x) .
$$

Since $f$ is a subexponential density, if $x_{0}$ is sufficiently large there must be a $\delta>0$ such that

$$
f^{* 2}(x) \geq(2+\delta) f(x) \quad \text { for all } x \geq x_{0}
$$

Hence,

$$
I_{2} \leq b_{1} b_{2}(2+\delta) f(x) .
$$

To conclude the proof, let $A=2 a+2+\delta$. 
Theorem 5.2. Let $F \in \mathcal{S}_{a c}$ and $f_{1}, \ldots, f_{d}$ be densities with $f_{i}(x) \sim c_{i} f(x)$ for some $c_{i} \geq 0, i=1, \ldots, d$. Then for any $\varepsilon>0$ there is a $D=D(\varepsilon)$ and $x_{0}=x_{0}(\varepsilon)$ such that

$$
f_{1}^{* n_{1}} * \cdots * f_{n_{d}}^{* n_{d}}(x) \leq D(1+\varepsilon) f(x)
$$

for any $x \geq x_{0}$ and $n_{1}, \ldots, n_{d} \in \mathbb{N}$. Furthermore

$$
f_{1}^{* n_{1}} * \cdots * f_{n_{d}}^{* n_{d}}(x) \sim\left(n_{1} c_{1}+\cdots+n_{d} c_{d}\right) f(x) .
$$

Proof. Analogous to Proposition 4.2.

\section{Proof of Theorem 2.2}

Recall that $\boldsymbol{P}=\left(p_{i j}\right)_{i, j \in \mathcal{E}}$ where the entries are defined by $p_{i j}=\left\|F_{i j}\right\|$. Let $\lambda=$ $\operatorname{spr}(\boldsymbol{P})$ be the Perron-Frobenius root of $\boldsymbol{P}$ and $\boldsymbol{v}$ the corresponding right eigenvector. Hence, $\boldsymbol{P}^{n} \boldsymbol{v}=\lambda^{n} \boldsymbol{v}$, which by strict positivity of $\boldsymbol{v}$ implies that the $n$ 'th element $p_{i j}^{(n)}$ of $\boldsymbol{P}^{n}$ will decay at rate $\lambda^{n}$. We will first treat the case where in addition to $\lambda<1$ the matrix $\boldsymbol{P}$ is substochastic. This means that the row sums satisfy $\sum_{j} p_{i j} \leq 1$, with strict inequality for at least one $i$.

Introduce an irreducible absorbing Markov chain $\left(\xi_{n}\right)_{n \in \mathbb{N}}$ with state space $\mathcal{E} \cup\{\dagger\}$ where $\dagger$ is the coffin state. Let $\boldsymbol{Q}=\left(q_{i j}\right)_{i, j \in \mathcal{E} \cup\{\dagger\}}$ be the transition matrix with $q_{i j}=p_{i j}$ for $i, j \in \mathcal{E}, q_{\dagger i}=0, q_{\dagger \dagger}=1$, and $q_{i \dagger}=1-\sum_{j \in \mathcal{E}} p_{i j}$. Recall that there must be at least one index $i$ such that $q_{i \dagger}>0$. Then $N=\inf \left\{n \geq 0: \xi_{n}=\dagger\right\}$ will be the time until absorbtion.

Let $T_{n}$ denote the waiting time between jump $n$ and $n+1$. Then $T_{0}, T_{1}, \ldots$ are conditionally independent given $\mathcal{F}=\sigma\left(\xi_{0}, \xi_{1}, \ldots\right)$ with conditional distribution function satisfying

$$
G_{i j}(t)=\mathbb{P}\left(T_{n} \leq t \mid \mathcal{F}\right)=\mathbb{P}\left(T_{n} \leq t \mid \xi_{n}, \xi_{n+1}\right)
$$

on $\left\{\xi_{n}=i, \xi_{n+1}=j\right\}$, and we have

$$
F_{i j}(t)=\mathbb{P}\left(\xi_{n+1}=j, T_{n} \leq t \mid \xi_{n}=i\right)=q_{i j} G_{i j}(t) .
$$

Henceforth we will only consider $i, j \neq \dagger$. Thus, $F_{i j}(t)=p_{i j} G_{i j}(t)$.

The solution of the Markov renewal equation (1.1) is given by Proposition 4.4 in [8] as

$$
Z_{i}(x)=\sum_{j \in \mathcal{E}} Z_{i j}(x) \quad \text { where } Z_{i j}(x)=\int_{0}^{x} z_{j}(x-y) U_{i j}(d y)
$$

with Markov renewal kernel $U_{i j}$ being the expected number of returns to state $j \in \mathcal{E}$ before time $t$ given that the Markov chain starts in state $i \in \mathcal{E}$. That is,

$$
U_{i j}(t)=\sum_{n=0}^{\infty}\left(\boldsymbol{F}^{* n}\right)_{i j}(t)=\sum_{n=0}^{\infty} \mathbb{P}_{i}\left(\xi_{n}=j, S_{n-1} \leq t\right),
$$

where $S_{n-1}=T_{0}+\cdots+T_{n-1}$. First, it is necessary to consider the local asymptotics of $U_{i j}$. 
Lemma 6.1. Under the assumptions of Theorem 2.2,

$$
U_{i j}(t+\Delta) \sim d_{i j} G(t+\Delta) .
$$

Proof. Notice that the $n$ 'th convolution of the semi-Markov kernel also can be defined locally

$$
\left(\boldsymbol{F}^{* n}\right)_{i j}(t+\Delta)=\mathbb{P}_{i}\left(\xi_{n}=j, S_{n-1} \in t+\Delta\right) .
$$

Rewriting the right-hand side using conditional expectations yields

$$
\begin{aligned}
\mathbb{P}_{i}\left(\xi_{n}\right. & \left.=j, S_{n-1} \in t+\Delta\right)=\mathbb{P}_{i}\left(\xi_{n}=j, T_{0}+\cdots+T_{n-1} \in t+\Delta\right) \\
& =p_{i j}^{(n)} \mathbb{P}\left(T_{0}+\cdots+T_{n-1} \in t+\Delta \mid \xi_{0}=i, \xi_{n}=j\right) \\
& =p_{i j}^{(n)} \mathbb{E}\left[\mathbb{E}\left[\mathbb{P}\left(T_{0}+\cdots+T_{n-1} \in t+\Delta \mid \xi_{0}=i, \xi_{n}=j\right) \mid \xi_{1}, \ldots, \xi_{n-1}\right]\right] \\
& =p_{i j}^{(n)} \mathbb{E}\left[G_{i j}^{(n)}(t+\Delta)\right],
\end{aligned}
$$

where $G_{i j}^{(n)}$ is the distribution of the sum $T_{0}+\cdots+T_{n-1}$ conditioned on the Markov states $\xi_{0}=i, \xi_{1}, \ldots, \xi_{n-1}, \xi_{n}=j$.

Let $N_{k \ell}^{(n)}$ the the random variable counting the number of jumps from state $k \in \mathcal{E}$ to state $\ell \in \mathcal{E}$ before the $n$ 'th jump, that is

$$
N_{k \ell}^{(n)}=\sum_{m=0}^{n-1} \mathbb{1}_{\left\{\xi_{m}=\ell, \xi_{m+1}=k\right\}} .
$$

Correspondingly, let $N_{k \ell \mid i j}^{(n)}$ be the random variable representing the number of jumps from $k \in \mathcal{E}$ to $\ell \in \mathcal{E}$ before jump $n$ given that $\xi_{0}=i$ and $\xi_{n}=j$, i.e. it is distributed as $N_{k \ell}^{(n)}$ conditioned on $\xi_{0}=i, \xi_{n}=j$. This has expected value

$$
\begin{aligned}
\mathbb{E}\left[N_{k \ell \mid i j}^{(n)}\right]=\mathbb{E}\left[N_{k \ell}^{(n)} \mid \xi_{0}=i, \xi_{n}=j\right]=\frac{\mathbb{E}\left[\sum_{m=0}^{n-1} \mathbb{1}_{\left\{\xi_{0}=i, \xi_{m}=k, \xi_{m+1}=\ell, \xi_{n}=j\right\}}\right]}{\mathbb{P}\left(\xi_{0}=i, \xi_{n}=j\right)} \\
=\frac{\sum_{m=0}^{n-1} \mathbb{P}\left(\xi_{0}=i, \xi_{m}=k, \xi_{m+1}=\ell, \xi_{n}=j\right)}{\mathbb{P}\left(\xi_{0}=i, \xi_{n}=j\right)}=\frac{\sum_{m=0}^{n-1} p_{i k}^{(m)} p_{k \ell} p_{\ell j}^{(n-m-1)}}{p_{i j}^{(n)}} \\
=\frac{\sum_{m=0}^{n-1}\left(\boldsymbol{P}^{m}\right)_{i k} p_{k \ell}\left(\boldsymbol{P}^{n-m-1}\right)_{\ell j}}{\left(\boldsymbol{P}^{n}\right)_{i j}}
\end{aligned}
$$

Recall that $G_{i j}^{(n)}$ is the distribution of the sum of the random variables $T_{0}, T_{1}$, $\ldots, T_{n-1}$ conditioned on the Markov chain until the $n$th jump. The distribution of $T_{m}$ merely depends on $\xi_{m}$ and $\xi_{m+1}$. Therefore, $G_{i j}^{(n)}$ can be interpreted as the random convolution

$$
G_{i j}^{(n)}(t+\Delta)=\underset{k, \ell \in \mathcal{E}}{*} G_{k \ell}^{* N_{k \ell \mid i j}^{(n)}}(t+\Delta) .
$$

Applying Theorem 4.3 the local asymptotics of $G_{i j}^{(n)}$ can then be specified as

$$
\mathbb{E}\left[\underset{k, \ell \in \mathcal{E}}{*} G_{k \ell}^{* N_{k \ell \mid i j}^{(n)}}(t+\Delta)\right] \sim\left(\sum_{k, \ell \in \mathcal{E}} \mathbb{E}\left[N_{k \ell \mid i j}^{(n)}\right] c_{k \ell}\right) G(t+\Delta) .
$$


This asymptotic specification transfers to the semi-Markov kernel as follows

$$
\left(\boldsymbol{F}^{* n}\right)_{i j}(t+\Delta) \sim \sum_{k, \ell \in \mathcal{E}} \sum_{m=0}^{n-1}\left(\boldsymbol{P}^{m}\right)_{i k} p_{k \ell}\left(\boldsymbol{P}^{n-m-1}\right)_{\ell j} c_{k \ell} G(t+\Delta)
$$

and on to the Markov renewal kernel

$$
\begin{aligned}
U_{i j}(t+\Delta) & \sim \sum_{n=0}^{\infty} \sum_{k, \ell \in \mathcal{E}} \sum_{m=0}^{n-1}\left(\boldsymbol{P}^{m}\right)_{i k} p_{k \ell}\left(\boldsymbol{P}^{n-m-1}\right)_{\ell j} c_{k \ell} G(t+\Delta) \\
& =\sum_{k, \ell \in \mathcal{E}} \sum_{m=0}^{\infty} \sum_{n=m+1}^{\infty}\left(\boldsymbol{P}^{m}\right)_{i k} p_{k \ell}\left(\boldsymbol{P}^{n-m-1}\right)_{\ell j} c_{k \ell} G(t+\Delta) .
\end{aligned}
$$

Since $\boldsymbol{P}$ has spectral radius strictly less than 1 the infinite series above converges with limits

$$
\sum_{n=m+1}^{\infty}\left(\boldsymbol{P}^{n-m-1}\right)_{\ell j}=\sum_{k=0}^{\infty}\left(\boldsymbol{P}^{k}\right)_{\ell j}=(\boldsymbol{I}-\boldsymbol{P})_{\ell j}^{-1}, \quad \sum_{m=0}^{\infty}\left(\boldsymbol{P}^{m}\right)_{i k}=(\boldsymbol{I}-\boldsymbol{P})_{i k}^{-1} .
$$

Thus

$$
U_{i j}(t+\Delta) \sim\left(\sum_{k, \ell \in \mathcal{E}}(\boldsymbol{I}-\boldsymbol{P})_{i k}^{-1} p_{k \ell}(\boldsymbol{I}-\boldsymbol{P})_{\ell j}^{-1} c_{k \ell}\right) G(t+\Delta),
$$

concluding the proof.

For the proof of Theorem 2.2, it suffices in view of (6.1) to find the asymptotics of the summands $Z_{i j}(x)=\int_{0}^{x} z_{j}(x-y) U_{i j}(d y)$ in the solution of the Markov renewal equation. As an introductory remark, notice that $G \in \mathcal{S}_{\Delta}$ for all $T>0$ has the implications

$$
G(x, x+1 / n] \sim \frac{g(x)}{n} \quad \text { for all } n \quad \text { and } \quad \frac{g(x+y)}{g(x)} \rightarrow 1 \quad \text { for all }|y|<y_{0}<\infty
$$

for some appropriate $y_{0}$. For a suitable $A<x / 2$ decompose $Z_{i j}(x)$ into three parts, namely

$$
\begin{aligned}
Z_{i j}(x) & =\left(\int_{0}^{A}+\int_{A}^{x-A}+\int_{x-A}^{x}\right) z_{j}(x-y) U_{i j}(\mathrm{~d} y) \\
& \equiv J_{1}(x, A)+J_{2}(x, A)+J_{3}(x, A) .
\end{aligned}
$$

We now consider and evaluate the three parts separately. In case i) of the Theorem we have

$$
\begin{aligned}
J_{1}(x, A) & =\int_{0}^{A} z_{j}(x-y) U_{i j}(\mathrm{~d} y) \\
& =g(x) \int_{0}^{A} \frac{z_{j}(x-y)}{g(x-y)} \frac{g(x-y)}{g(x)} U_{i j}(\mathrm{~d} y)=o(g(x))
\end{aligned}
$$


when $x \rightarrow \infty$. Also

$$
\begin{aligned}
J_{2}(x, A) & =\int_{A}^{x-A} z_{j}(x-y) U_{i j}(\mathrm{~d} y)=\int_{A}^{x-A} \frac{z_{j}(x-y)}{g(x-y)} g(x-y) U_{i j}(\mathrm{~d} y) \\
& =o(1) \int_{A}^{x-A} g(x-y) U_{i j}(\mathrm{~d} y)=o(g(x)) \int_{A}^{x-A} \frac{g(x-y)}{g(x)} U_{i j}(\mathrm{~d} y) .
\end{aligned}
$$

From this we can conclude

$$
\lim _{A \rightarrow \infty} \lim _{x \rightarrow \infty} \frac{J_{2}(x, A)}{g(x)}=\lim _{A \rightarrow \infty} \lim _{x \rightarrow \infty} \frac{o(g(x))}{g(x)}(U(x-A)-U(A))=0 .
$$

Finally, consider a finite partition of the interval $(x-A, x]$ into $n$ equally sized intervals. $A$ is now assumed to be an integer. Furthermore, let

$$
\bar{z}_{n}(x)=\sup _{|y-x| \leq \frac{1}{n}} z(y) .
$$

Then we can bound $J_{3}$ from above by the upper Riemann sum

$$
\begin{aligned}
J_{3}(x, A) & =\int_{x-A}^{x} z_{j}(x-y) U_{i j}(\mathrm{~d} y) \leq \sum_{k=0}^{A n-1} \bar{z}_{n}\left(\frac{k}{n}\right) U_{i j}\left(x-\frac{k+1}{n}, x-\frac{k}{n}\right) \\
& \sim d_{i j} \sum_{k=0}^{A n-1} \bar{z}_{n}\left(\frac{k}{n}\right) G\left(x-\frac{k+1}{n}, x-\frac{k}{n}\right) \\
& \sim g(x) \frac{d_{i j}}{n} \sum_{k=0}^{A n-1} \bar{z}_{n}\left(\frac{k}{n}\right) \sim g(x) \frac{d_{i j}}{n} \sum_{k=0}^{\infty} \bar{z}_{n}\left(\frac{k}{n}\right)
\end{aligned}
$$

as $x \rightarrow \infty$ and $A \rightarrow \infty$. Since $z$ is assumed to be directly Riemann integrable, we have

$$
\frac{1}{n} \sum_{k=0}^{\infty} \bar{z}_{n}\left(\frac{k}{n}\right) \rightarrow I_{j}
$$

as $n$ tends to infinity. So we have now obtained

$$
\limsup _{A \rightarrow \infty} \limsup _{x \rightarrow \infty} \frac{J_{3}(x, A)}{g(x)} \leq d_{i j} I_{j}
$$

Using the same approach with lim inf a similar bound from below is obtained using lower Riemann sums. This finishes case i).

Now consider case ii). The first of the decomposed parts has the asymptotics

$$
\begin{aligned}
J_{1}(x, A) & =\int_{0}^{A} z_{j}(x-y) U_{i j}(\mathrm{~d} y) \sim a_{j} g(x) \int_{0}^{A} \frac{g(x-y)}{g(x)} U_{i j}(\mathrm{~d} y) \\
& \sim a_{j} g(x) U_{i j}(A)
\end{aligned}
$$

which leads to

$$
\lim _{A \rightarrow \infty} \lim _{x \rightarrow \infty} \frac{J_{1}(x, A)}{g(x)}=\lim _{A \rightarrow \infty} \lim _{x \rightarrow \infty} a_{j} U_{i j}(A)=k_{i j} a_{j} .
$$


The second part in case ii) can also be evaluated as follows

$$
\begin{aligned}
J_{2}(x, A) & =\int_{A}^{x-A} z_{j}(x-y) U_{i j}(\mathrm{~d} y)=\int_{A}^{x-A} \frac{z_{j}(x-y)}{g(x-y)} g(x-y) U_{i j}(\mathrm{~d} y) \\
& =O(1) \int_{A}^{x-A} g(x-y) U_{i j}(\mathrm{~d} y) .
\end{aligned}
$$

Notice the similarity to $J_{2}$ in case i). Corresponding calculations show that $J_{2}(x, A)=$ $o(g(x)) . J_{3}$ is also similar to case i), which concludes case ii).

In case iii) we are no longer able to consider the decomposition we have so far. Instead, let $K_{j}$ denote the probability measure with density $z_{j}(x) / I_{j}$. Recall that $K_{j} \in \mathcal{S}_{\Delta}$. Now let $\Delta=(0,1]$ and consider a new decomposition

$$
\begin{aligned}
\int_{0}^{x} z_{j}(x-y) U_{i j}(\mathrm{~d} y) & =\int_{0}^{x-A} z_{j}(x-y) U_{i j}(\mathrm{~d} y)+\int_{x-A}^{x} z_{j}(x-y) U_{i j}(\mathrm{~d} y) \\
& \equiv I_{1}(x, A)+I_{2}(x, A),
\end{aligned}
$$

where the second term satisfies

$$
I_{2}(x, A) \leq A \sup _{y \leq A}\left|z_{j}(y)\right| U_{i j}(x-A, x]=o\left(z_{j}(x)\right) .
$$

Letting $A$ tend to infinity in a slower rate than $x$ (recall that we have chosen $A$ to be less than $x / 2$ ) will preserve this inequaility. Now consider a corresponding decomposition of the convolution $K_{j} * U_{i j}$.

$$
\begin{aligned}
\left(K_{j} * U_{i j}\right)(x+\Delta) & =\int_{0}^{x} K_{j}(x-y+\Delta) U_{i j}(\mathrm{~d} y) \\
& =\int_{0}^{x-A} K_{j}(x-y+\Delta) U_{i j}(\mathrm{~d} y)+\int_{x-A}^{x} K_{j}(x-y+\Delta) U_{i j}(\mathrm{~d} y) \\
& \equiv I_{1}^{\prime}(x, A)+I_{2}^{\prime}(x, A) .
\end{aligned}
$$

As for $I_{2}$ we can evaluate $I_{2}^{\prime}(x, A)=o\left(z_{j}(x)\right)$. Since $z_{j} \in \mathcal{L}$ we have $z_{j}(x) \sim I_{j}$. $K_{j}(x+\Delta)$ for $\Delta=(0,1]$ and therefore $I_{1}(x, A) \sim I_{j} \cdot I_{1}^{\prime}(x, A)$. This yields

$$
\int_{0}^{x} z_{j}(x-y) U_{i j}(\mathrm{~d} y) \sim I_{j} \cdot\left(K_{j} * U_{i j}\right)(X+\Delta) .
$$

Due to the assumption $z_{j}(x) / g(x) \rightarrow \infty$ the use of Proposition 3 in [9] with $c_{1}=1$ and $c_{2}=0$ gives

$$
I_{j} \cdot\left(K_{j} * U_{i j}\right)(x+\Delta) \sim k_{i j} I_{j} K(x+\Delta) \sim k_{i j} z_{j}(x)
$$

which concludes the proof of Theorem 2.2 in the substochastic case.

If instead of $\boldsymbol{P}$ being substochastic it merely satisfies $\operatorname{spr}(\boldsymbol{P})<1$, we define the measure $\tilde{F}_{i j}(d x)=F_{i j}(d x) v_{i} / v_{j}$ for $i, j \in \mathcal{E}$ and let $\tilde{\boldsymbol{P}}$ be the matrix with elements $\left\|\tilde{F}_{i j}\right\|=\left\|F_{i j}\right\| v_{i} / v_{j}$. $\tilde{\boldsymbol{P}}$ will then be a substochastic matrix with row sums $\lambda$ and 
therefore it must also have spectral radius less than one. Letting $\tilde{Z}_{i}(x)=v_{i} Z_{i}(x)$ and $\tilde{z}_{i}(x)=v_{i} z_{i}(x)$ another renewal equation occurs

$$
\tilde{Z}_{i}(x)=\tilde{z}_{i}(x)+\sum_{j \in \mathcal{E}} \int_{0}^{x} \tilde{Z}_{j}(x-y) \tilde{F}_{i j}(d y),
$$

which has the same properties as analyzed previously in the substochastic case with Markov kernel $\tilde{U}_{i j}=U_{i j} v_{i} / v_{j}$. What we have shown already can therefore be applied with coefficients $\tilde{a}_{j}, \tilde{d}_{i j}, \tilde{k}_{i j}$ and $\tilde{I}_{j}$. The assumption of the asymptotic properties of $G_{i j}$ stated in Theorem 2.2 implies that $\tilde{G}_{i j}(t+\Delta)=v_{i} G_{i j}(t+\Delta) / v_{j} \sim \tilde{c}_{i j} G(t+\Delta)$, where $\tilde{c}_{i j}=c_{i j} v_{i} / v_{j}$ and same relation transfers to $\tilde{d}_{i j}=d_{i j} v_{i} / v_{j}$. Correspondingly for $\tilde{k}_{i j}=\tilde{U}_{i j}(0, \infty)=U_{i j}(0, \infty) v_{i} / v_{j}=k_{i j} v_{i} / v_{j}$. Last to mention is $\tilde{I}_{j}=\int_{0}^{\infty} \tilde{z}_{j}(y) d y=$ $v_{j} \int_{0}^{\infty} z_{j}(y) d y=v_{j} I_{j}$ and $\tilde{a}_{j}=v_{j} a_{j}$. Inserting these into the asympotics of $\tilde{Z}_{i}$ gives the same result as Theorem 2.2. For example in case ii), the substochatic results gives $\tilde{Z}_{i}(x) \sim \sum_{j \in \mathcal{E}}\left(\tilde{I}_{j} \tilde{d}_{i j}+\tilde{a}_{j} \tilde{k}_{i j}\right) g(x)$ which translates to

$$
\begin{aligned}
Z_{i}(x)=\frac{1}{v_{i}} \tilde{Z}_{i}(x) & \sim \frac{1}{v_{i}} \sum_{j \in \mathcal{E}}\left(v_{j} I_{j} \frac{v_{i}}{v_{j}} d_{i j}+v_{j} a_{j} \frac{v_{i}}{v_{j}} k_{i j}\right) g(x) \\
& =\sum_{j \in \mathcal{E}}\left(I_{j} d_{i j}+a_{j} k_{i j}\right) g(x) .
\end{aligned}
$$

\section{References}

[1] P. Embrechts, C. Klüppelberg, and T. Mikosch, Modelling Extremal Events for Insurance and Finance, vol. 33. Springer Science, 1997.

[2] R. Adler, R. Feldman, and M. Taqqu, A Practical Guide to Heavy Tails. Birkhäuser, Basel, 1998.

[3] R. Barakat, "Sums of independent lognormally distributed random variables," JOSA, vol. 66, no. 3, pp. 211-216, 1976.

[4] X. Cao, Inference for Age-dependent Branching Process and Their Applications. PhD thesis, George Mason University, 2015.

[5] S. I. Resnick, Heavy-tail Phenomena: Probabilistic and Statistical Modeling. Springer Science \& Business Media, 2007.

[6] P. Jagers, Branching Processes with Biological Applications. Wiley, 1975.

[7] W. Feller, An Introduction to Probability Theory and Its Applications. Volume II. John Wiley \& Sons London-New York-Sydney-Toronto, 2nd ed., 1971.

[8] S. Asmussen, Applied Probability and Queues. Springer, 2nd ed., 2003.

[9] S. Asmussen, S. Foss, and D. Korshunov, "Asymptotics for sums of random variables with local subexponential behaviour," Journal of Theoretical Probability, vol. 16, no. 2, pp. 489-518, 2003.

[10] K. Wang, Y. Chen, and Z. Tan, "Asymptotics for the solutions to defective renewal equations," Abstract and Applied Analysis, vol. 2014, pp. 1-5, 2014. 
[11] S. Asmussen, L. Lipsky, and S. Thompson, "Markov renewal methods in restart problems in complex systems," in The Fascination of Probability, Statistics and their Applications, pp. 501-527, Springer, 2016.

[12] S. Foss, D. Korshunov, and S. Zachary, An Introduction to Heavy-tailed and Subexponential Distributions. Springer, 2011.

[13] K. B. Athreya and P. E. Ney, Branching Processes. Springer, 1972. 